\title{
Evaluation of the Mechanism of Modified Lingguizhugan Decoction in the Treatment of Nonalcoholic Fatty Liver Disease
}

\author{
Fuyuan Yang $\mathbb{D}^{1},{ }^{1}$ Jianxiang Zhou, ${ }^{2}$ Bo Su, ${ }^{3}$ Qiong Zhang, ${ }^{3}$ Xuezhi Luo, ${ }^{4}$ Fei Wang, \\ Jiangrong Huang $\mathbb{D},{ }^{1}$ and Wei Huang $\mathbb{D}^{2}$ \\ ${ }^{1}$ School of Basic Medicine, Yangtze University Health Science Center, Jingzhou, Hubei 434020, China \\ ${ }^{2}$ Department of Endocrinology, Jingzhou Hospital of Traditional Chinese Medicine, \\ Third Clinical Medical College, Yangtze University, Jingzhou, Hubei 434020, China \\ ${ }^{3}$ School of Basic Medicine, Yangtze University Health Science Center, Jingzhou, Hubei 434020, China \\ ${ }^{4}$ Hubei College of Chinese Medicine, Jingzhou, Hubei 434020, China \\ Correspondence should be addressed to Jiangrong Huang; huangjr999@sina.com and Wei Huang; huangweiiiiii@126.com
}

Received 29 April 2021; Revised 26 October 2021; Accepted 2 December 2021; Published 25 January 2022

Academic Editor: Waseem Hassan

Copyright ( 2022 Fuyuan Yang et al. This is an open access article distributed under the Creative Commons Attribution License, which permits unrestricted use, distribution, and reproduction in any medium, provided the original work is properly cited.

\begin{abstract}
Nonalcoholic steatohepatitis (NASH) may develop into cirrhosis and liver cancer, which imposes a great burden to individuals and society. Lingguizhugan decoction is a commonly used dampness dispelling medication in traditional Chinese medicine and is often used to treat those with phlegm and retained fluid from various causes and pathogeneses. The objective of this study was to explore the effect and mechanism of modified Lingguizhugan decoction (MLGZG) on lipid metabolism and the inflammatory response to identify a theoretical basis to promote its clinical application in NASH therapy. After treatment with MLGZG for 8 weeks, the weight of high-fat-diet (HFD)-fed NASH rats was significantly higher than that of rats in the normal group, and the weights in each dose group were significantly lower than those in the model group. The treatment groups (low, medium, and high doses) had different degrees of improvement in the changes in hepatocyte tissue structure, steatosis, and inflammatory infiltration. Compared with that in the normal group, the expression of TNF receptor-associated factor-3 (TRAF-3) and nuclear factor $\kappa \mathrm{B}$ $(\mathrm{NF} \kappa \mathrm{B})$ in the model group significantly increased to varying degrees. Compared with the NASH group, the treatment groups (low, middle, and high doses) showed modified lipid metabolism gene expression and decreased inflammatory factor expression levels. Modified Lingguizhugan decoction can improve the general condition of rats with nonalcoholic fatty liver disease by reducing the expression levels of TRAF3, NF- $\kappa \mathrm{B}$, the Toll-like receptor 4 (TLR-4) pathway, and related proteins, as well as the expression levels of lipid metabolism genes and cytokines.
\end{abstract}

\section{Introduction}

Nonalcoholic steatohepatitis (NASH) is the exacerbated form of nonalcoholic fatty liver disease. While the etiology and pathogenic mechanisms underlying NASH remain largely elusive, the development of NASH results from hepatocyte damage, inflammation, and tissue destruction in the liver, leading to metabolic system and circulatory system dysfunction. Thus, NASH is a great threat to human health. Despite enormous efforts to optimize existing drugs and develop new drugs in recent years, a large portion of NASH patients are still not responsive to medical treatment. The chronic inflammatory response has been shown to contribute to the development of NASH. Genetic factors and abnormal nutritional metabolism lead to the accumulation of adipose tissue in obese patients. Toll-like receptor 4 (TLR4) can be activated by saturated fatty acids and then upregulates the release of proinflammatory factors such as tumor necrosis factor- $\alpha$ (TNF- $\alpha$ ), interleukin-6 (IL-6), and monocyte chemoattractant protein-1 (MCP-1), leading to the occurrence of a chronic inflammatory response [1-3]. Then, insulin resistance is caused by nuclear factor $\kappa \mathrm{B}$ (NF$\kappa \mathrm{B})$ and C-Jun N-terminal kinase (JNK) [4]. The accumulation of CD8 T cells mediated by inflammatory factors in 
obese patients enhances insulin resistance through the mislocation of glucose transporter type 2 (GLUT-2) [5]. Furthermore, insulin resistance and increased circulatingfree fatty acids cause intrahepatic fat accumulation, which, combined with chronic inflammatory response-induced cytotoxicity, results in damage to liver cells and an overactive inflammatory response [6].

In multiple cell types, including hepatocytes, cardiomyocytes, osteoclasts, microglia, and inflammatory cells, TRAF3 is expressed and plays a key role in the regulation of inflammation [7-10]. Animal and clinical evidence indicates that the function and expression of TRAF3 are involved in the pathogenesis of a variety of inflammation-related diseases, such as inflammatory bowel disease, liver inflammation, diabetes, bone inflammation, encephalitis, and autoimmune inflammation. TRAF3 serves as an E3 ubiquitination signal transducer in inflammatory responses and is activated by TNF receptor (TNF-R) superfamily receptors, Toll-like receptors (TLRs), NOD-like receptors (NLRs), retinoic acid-inducible gene I (RIG-I), and cytokines $[11,12]$. TRAF3 expression is significantly increased in the liver of obese mice, and hepatocyte-specific TRAF3 knockout can alleviate insulin resistance. TRAF3 leads to transforming growth factor beta-activated kinase 1 (TAK1) ubiquitination and autophosphorylation in high-fat diet-fed mice and then activates the NF- $\kappa$ B inflammatory pathway and JNK-IRS1 (insulin receptor substrates 1) insulin resistance [13].

The traditional classic prescription Lingguizhugan decoction, originally recorded in Shanghan Zabing Lun, has been commonly used for the treatment of dizziness and palpitations. Based on years of clinical NASH treatment experience, we previously formulated a modified Lingguizhugan decoction (MLGZG) by adding herbs to the original prescription and achieved satisfactory efficacy in the treatment of obesity, insulin resistance, and other metabolic diseases, but the underlying mechanism of this effect is still unclear [14]. Modified Lingguizhugan decoction has many advantages in metabolic disease therapy [15]. It has been reported that Lingguizhugan decoction could inhibit TNF- $\alpha$ synthesis in the treatment of chronic heart failure. In NASH patients, the expression of the TNF- $\alpha$ gene and the inflammatory response were effectively reduced by Lingguizhugan decoction [16]. Our previous animal study demonstrated that MLGZG reduced blood glucose, fat, body weight, and insulin resistance in HFD rats. In addition, MLGZG was able to ameliorate obesity in rats with metabolic syndrome and reduce fat cell volume in obese rats [17]. Given that therapeutic strategies for NASH largely contribute to immune and inflammation modulation, we undertook the present study to check whether MLGZG can regulate inflammatory reactions through animal experiments and to explore the signaling pathways underlying the effect. After administration of MLGZG to an HFD-fed rat model of NASH, we detected the effects on serum lipids, inflammation levels, and the TNF $\alpha$-TRAF3-related signaling regulation pathway in model animals. MLGZG may serve as a novel mechanism for the treatment of NASH, as decreasing bile acid (BA) and farnesoid $X$ receptor (FXR) signaling may yield metabolic benefits $[8,14-19]$.

\section{Materials and Methods}

2.1. Preparation of $M L G Z G$. MLGZG is composed of 12 grams (g) Cortex Poria, 9 g Ramulus Cinnamomi, $12 \mathrm{~g}$ Rhizoma Atractylodis Macrocephalae, $12 \mathrm{~g}$ Radix Codonopsis, $12 \mathrm{~g}$ Rhizoma Pinelliae, $30 \mathrm{~g}$ Fructus Crataegi, $15 \mathrm{~g}$ Herba Siegesbeckiae, $9 \mathrm{~g}$ Carthamus tinctorius, $9 \mathrm{~g}$ Ligusticum wallichii, $15 \mathrm{~g}$ Radix Polygonum Multiflorum Preparata, and $6 \mathrm{~g}$ honey-fried licorice root. These components were soaked in $1125 \mathrm{ml}$ distilled water for $30 \mathrm{~min}$, followed by $40 \mathrm{~min}$ of boiling at $100{ }^{\circ} \mathrm{C}$. Then, $846 \mathrm{ml}$ distilled water was added and boiled for $30 \mathrm{~min}$, the liquid was collected, and the two doses were mixed. The decoction was then condensed to $1 \mathrm{ml}$ and stored at $4{ }^{\circ} \mathrm{C}$. Before use, it was warmed to $37{ }^{\circ} \mathrm{C}$ before use.

2.2. Quantitative Real-Time ( $q R T$ ) PCR. Total RNA was isolated using TRIzol Reagent (Invitrogen, Waltham, MA, USA). For reverse transcription, 1-5 mg total RNA was used, following the methods of Yang et al., 2018 [20]. First-strand complementary DNA synthesis was performed using oligo(T)18 and M-MLV enzyme (Promega, Madison, WI, USA). A PCR system was applied to amplify target genes (thermal cycling program: $1: 94{ }^{\circ} \mathrm{C} 4$ minutes (min); $2: 94{ }^{\circ} \mathrm{C}$ 30 seconds (s); $3: 55{ }^{\circ} \mathrm{C} 30 \mathrm{~s} ; 5: 72{ }^{\circ} \mathrm{C} 30 \mathrm{~s}$; and $4: 72{ }^{\circ} \mathrm{C}$ $10 \mathrm{~min}$; steps $2-5$ were repeated for $30-35$ cycles). The mRNA levels were measured by real-time PCR (Applied Biosystems) using SYBR Green Master Mix (Applied Biosystems). The total amount of mRNA was normalized to endogenous glyceraldehyde 3-phosphate dehydrogenase mRNA. The sequences of the primers are shown in Table 1.

2.3. Western Blot. Tissues were lysed at $4^{\circ} \mathrm{C}$ using radioimmunoprecipitation assay lysis buffer (RIPA, Thermo Fisher, Waltham, MA, USA) with a protease inhibitor cocktail for $30 \mathrm{~min}$. Supernatants were collected, and the protein concentrations were measured by a Bradford protein assay system (Bio-Rad, Hercules, CA, USA). Proteins were incubated with primary antibodies overnight at $4^{\circ} \mathrm{C}$, washed and incubated with horseradish peroxidase-conjugated secondary antibodies diluted $1: 10,000$ in $2 \%$ milk in Trisbuffered saline Tween-20. Antibodies used in the Western blot are listed in Table 2. The membranes were washed, and protein bands were detected with enhanced chemiluminescence reagent (Amersham, Little Chalfont, UK) and a ChemDoc ${ }^{\mathrm{TM}}$ XRS + system (Bio-Rad, Hercules, CA, USA) [21]. For quantification, densitometry in ImageJ was applied to quantify the relative band intensities.

2.4. HFD NASH Rat Model. Mature male Wistar rats (weight 300-350 g, purchased from Hubei Provincial Laboratory Animal Center) were used in this study. All animal experiments were approved by the Institutional Animal Care and Use Committee of Yangtze University (CJYXBEC2018-113, 2018.11.11) and were conducted in accordance with the NIH guidelines and regulations on animal experimentation. Rats were fed a high-fat diet (fat provides $60 \%$ of the total energy) 
Table 1: Primer list.

\begin{tabular}{|c|c|c|}
\hline Target & Sequence & Length \\
\hline GAPDH & $\begin{array}{l}\text { 5' AGTGCCAGCCTCGTCTCATA 3' } \\
\text { 3' GACTGTGCCGTTGAACTTGC 5' }\end{array}$ & $252 b p$ \\
\hline SREBP & $\begin{array}{l}\text { 5’ GTTAACGTGGGTCTCCTCCG 3' } \\
\text { 3' CCAGCATAGGGGGCATCAAA 5' }\end{array}$ & $168 \mathrm{bp}$ \\
\hline Leptin & $\begin{array}{l}\text { 5’ GGTGGCTGGTTTGTTTCTGT 3' } \\
\text { 3’ TATGTGGCTGCAGAGGTGAG 5' }\end{array}$ & $249 b p$ \\
\hline TNF- $\alpha$ & $\begin{array}{l}\text { 5' GATCTCAAAGACAACCAACTG 3' } \\
\text { 3' CTGGTATGAAATGGCAAATCG 5' }\end{array}$ & $138 \mathrm{bp}$ \\
\hline IL- $1 \beta$ & $\begin{array}{l}\text { 5' CCTTGTGCAAGTGTCTGAAGC 3' } \\
\text { 3' CCCAAGTCAAGGGCTTGGAA 5' }\end{array}$ & $147 \mathrm{bp}$ \\
\hline IL-10 & $\begin{array}{l}\text { 5’ CATTATGTGGGCACTGGCCT 3' } \\
\text { 3' GTAGATGCCGGGTGGTTCAA 5' }\end{array}$ & $156 \mathrm{bp}$ \\
\hline IL-12 & $\begin{array}{l}\text { 5' CCCATGGATCTTCGGGCAAG 3' } \\
\text { 3' TCACGGACCTGCTCACCTAC 5' }\end{array}$ & $283 \mathrm{bp}$ \\
\hline
\end{tabular}

Table 2: Antibody list.

\begin{tabular}{lcc}
\hline Antibody & Cat. no. & Company \\
\hline TLR-4 & AB22048 & Abcam \\
TRAF-3 & SC-6933 & Santa Cruz \\
NF- $\kappa$ b p65 & 8242 & Cell Signaling Technology \\
P-p65 & 3033 & Cell Signaling Technology \\
$\beta$-actin (42KD) & 4970 & Cell Signaling Technology \\
\hline
\end{tabular}

for 12 consecutive weeks to establish the model. The weights of the rats were measured every 2 days throughout the whole experiment [22]. Rats were randomly divided into five groups: a blank group, a NASH group, an MLGZG low-dose group, an MLGZG moderate-dose group, and an MLGZG high-dose group. MLGZG (6 g/(kg•day), $12 \mathrm{~g} /(\mathrm{kg} \bullet$ day), and $18 \mathrm{~g} /(\mathrm{kg} \bullet$ day)) was gavaged to rats in the MLGZG low-dose, MLGZG moderate-dose, and MLGZG high-dose groups. Rats in the control and NASH groups were gavaged with saline. At different time points, rats were sacrificed, and their livers were collected for further studies.

\subsection{Hematoxylin and Eosin (H\&E) Staining.} Paraffin-embedded colons were prepared in $6-\mu \mathrm{m}$ sections, deparaffinized using xylene and rehydrated through gradient alcohol $(100 \% * 2,95 \% * 2,80 \% * 1,70 \% * 1$, water- 1 , 90 s per step with shaking). H\&E staining was performed as previously described [20]. After rehydration, sections were stained with hematoxylin for $5 \mathrm{~min}$ and differentiated in acid ethanol $(0.36 \mathrm{~g} \mathrm{HCl}$ in $100 \mathrm{ml}$ ethanol, $\mathrm{pH}=1)$ for $3 \mathrm{~min}$. Then, the sections were washed in running tap water for $2 \mathrm{~min}$, blued in tap water for $10 \mathrm{~min}$, and stained in eosin $\mathrm{Y}$ solution for $1-2 \mathrm{~min}$. Then, $95 \% * 2$ plus $100 \% * 2$ alcohol was applied for dehydration, after which the specimens were cleared with xylene twice and finally mounted with histological mounting medium.

2.6. HFD Cell Model ELISA. After 7 days of housing and feeding, 24 male Sprague Dawley rats were randomly divided into 4 groups (Normal, MLGZG ${ }^{\mathrm{L}}, \mathrm{MLGZG}^{\mathrm{M}}$, and MLGZG $^{\mathrm{H}}$; 6 for each group), and saline or MLGZG was gavaged into the rats twice a day for 7 consecutive days. Then, drug-containing serum was collected as described in a previous study. Briefly, blood was collected from the abdominal aorta, quiesced for $1 \mathrm{~h}$, and centrifuged at 3000 RPM for $15 \mathrm{~min}$. The serum was collected and inactivated at $56{ }^{\circ} \mathrm{C}$ for $30 \mathrm{~min}$ and stored at $-80{ }^{\circ} \mathrm{C}$ for future use [23]. HEPG 2 cells were cultured in $37{ }^{\circ} \mathrm{C}$ incubators with $5 \% \mathrm{CO}_{2}$ and DMEM containing 10\% FBS and 1\% penicillamine. After $12 \mathrm{~h}$ of starvation with DMEM without serum, the HEPG2 cells were cultured with $0.1 \mathrm{mM}$ palmitic acid in complete culture medium and cultured for $24 \mathrm{~h}$ to establish the HFD cell model. After that, the cells were treated with $10 \%$ normal rat serum or drug-containing serum, and the cell supernatant was collected $24 \mathrm{~h}$ later for ELISA tests. The levels of TNF- $\alpha$, IL- $1 \beta$, and IL- 2 in the supernatant were tested with ELISA kits from Shanghai Jing Anti-Biology Company.

2.7. Serum Lipid Analysis. After feeding for 12 weeks, rats from the control, HFD, and MLGZG treatment groups were subjected to blood biochemical analysis. An automated biochemical analyzer (Type AU5421, Olympus, Japan) was used to estimate the concentrations of triglycerides (TGs), total cholesterol (TC), low-density lipoprotein cholesterol (LDL-C), and high-density lipoprotein cholesterol (HDL-C) in plasma [24].

2.8. Statistical Analysis. All experiments were repeated at least three times, and the data are presented as the mean\pm SEM. Student's $t$-test was applied to calculate the differences between two groups, while one-way analysis of variance (ANOVA) was applied for comparisons among three groups, and post hoc analyses were performed with the Newman-Keuls multiple comparison test. Statistical significance was considered at $P<0.05$ (\# indicates $P<0.05$, \#\# indicates $P<0.01$, and \#\#\# indicates $P<0.001$ HFD group compared to the normal control group; * indicates $P<0.05$, ** indicates $P<0.01$, and ${ }^{* * *}$ indicates $P<0.001$ MLGZGtreated groups compared to HFD group). Statistical calculations were performed with GraphPad Prism software.

\section{Results}

\subsection{MLGZG Ameliorates NASH in a HFD NASH Rat Model.} In this study, to test the potential therapeutic effect of MLGZG on NASH, we used an HFD-induced NASH rat model. In this model, fed on a high-fat diet, rats presented the most evident clinical signs of NASH from week 5 to week 12 , along with obvious abdominal obesity, significant weight gain in the early stage, and a tendency for weight decline in the later stage. MLGZG demonstrated a more pronounced effect on the reduction in weight at week 12 (Figure 1(a)). Meanwhile, the liver mass of HFD rats was significantly increased relative to that of normal rats, and MLGZG feeding effectively reduced the liver mass (Figure 1(b)). In MLGZG-fed HFD rats, the liver index was also significantly 


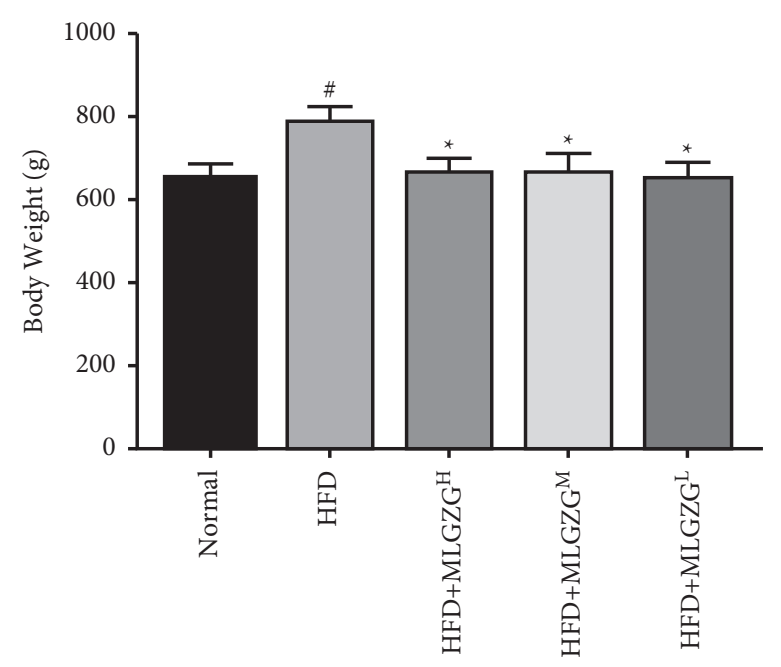

(A)

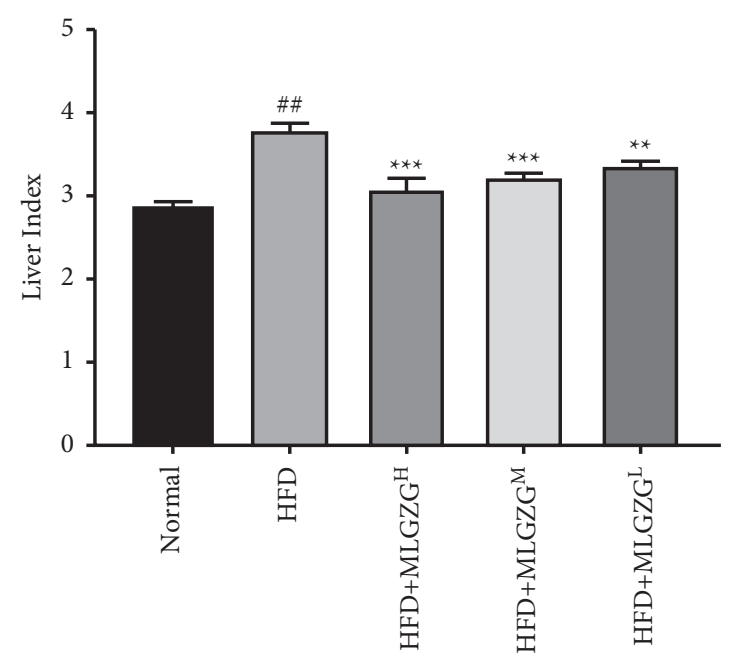

(B)

FIGURE 1: Final body mass of the rats at week 12 (A) and liver index of the rats at week 12 (B). (HFD: high-fat diet; MLGZG : MLGZG low dose; MLGZG ${ }^{\mathrm{M}}$ : MLGZG moderate dose; MLGZG ${ }^{\mathrm{H}}$ : MLGZG high dose. \# indicates $P<0.05$, \#\# indicates $P<0.01$, and \#\#\# indicates $P<0.001$, HFD group compared to the normal control group; ${ }^{*}$ indicates $P<0.05,{ }^{* *}$ indicates, $P<0.01$ and ${ }^{* * *}$ indicates $P<0.001$, MLGZG-treated groups compared to the HFD group).

reduced compared with that in HFD rats. Histopathological examination of the liver sections showed that MLGZG significantly reduced the extension and severity of the inflamed area and the damage to hepatocytes compared with that of HFD-only rats. H\&E staining indicated that MLGZG effectively reduced hepatic lobule structure damage, disordered cell arrangement, and the number of lipid vesicles (Figure 2). These results suggest that MLGZG administration reduces NASH development and liver damage.

\subsection{MLGZG Modulates Blood Lipids through Lipid Meta-} bolism Genes. The blood lipid content was detected, and HFD rats had a marked increase in blood lipid content relative to the control group (Table 3). Except for TG content, the TC and LDL-C contents were largely increased in HFD rats relative to control rats, and treatment with MLGZG significantly decreased the TG, TC, and LDL contents. To further characterize the therapeutic identities of MLGZG, we used real-time PCR. As shown in Figure 3, lipid metabolism genes were differentially expressed between MLGZG-fed NASH rats and NASH rats. The expression levels of SREBP and leptin were significantly downregulated by MLGZG feeding, indicating that MLGZG alters the lipid genesis of NASH rats (Figure 3).

\subsection{MLGZG Suppresses the Immune Response during NASH.} Interestingly, our Western blot results revealed that compared with that of HFD rats, the expression of NF- $\kappa$ B P-p65 was significantly decreased in MLGZG-treated rats, and we also found that TLR-4 and TRAF3 expression levels were significantly reduced by MLGZG feeding. Since activation of the TLR-4/TRAF3/NF- $\kappa$ B pathway plays a critical role in the hepatic inflammatory response, it is plausible that the therapeutic effect of MLGZG is mediated by the TLR-4/
TRAF3/NF- $\kappa$ B pathway (Figure 4 ). We also determined whether MLGZG was able to modulate the hepatic inflammatory reaction. We showed that MLGZG dramatically suppressed the mRNA expression of proinflammatory cytokines, such as TNF- $\alpha$ and IL-12. Since IL-10 is an antiinflammatory cytokine and immunosuppressive factor, we also determined the effect of MLGZG on IL-10 expression. Interestingly, MLGZG significantly decreased the mRNA expression of IL-10 in HFD rat livers (Figure 5). To check the inflammatory regulatory function of MLGZG in vitro, we treated HFD HEPG2 cells with drug-containing serum. The ELISA results showed that the cytokine level in culture medium was increased by palmitic acid, and MLGZGcontaining serum significantly reduced TNF- $\alpha$, IL- $1 \beta$, and IL-2 secretion by HFD HEPG2 cells (Figure 6).

\section{Discussion}

MLGZG is a well-established Chinese medicine prescription. This is the first study to explore the mechanism of action of MLGZG in NASH from the perspective of TRAF3-related pathways, which can provide an experimental basis for the development of an MLGZG-based NASH treatment. HFDinduced NASH is the most commonly used animal model for the study of human NASH [25]. In our study, by orally feeding rats a high-fat diet for 12 weeks, we successfully established a NASH rat model that recapitulates the early, middle, and late stages of NASH pathology. With respect to the NASH group, MLGZG appeared to offer evident therapeutic advantages in NASH treatment. First, MLGZG led to a prominent reduction in body weight (Figure 1). Second, MLGZG resulted in profound improvements in HFD-induced NASH pathology; for example, hepatocyte damage and the inflammatory response were significantly alleviated in MLGZG-treated rat livers (Figure 2). Third, the 

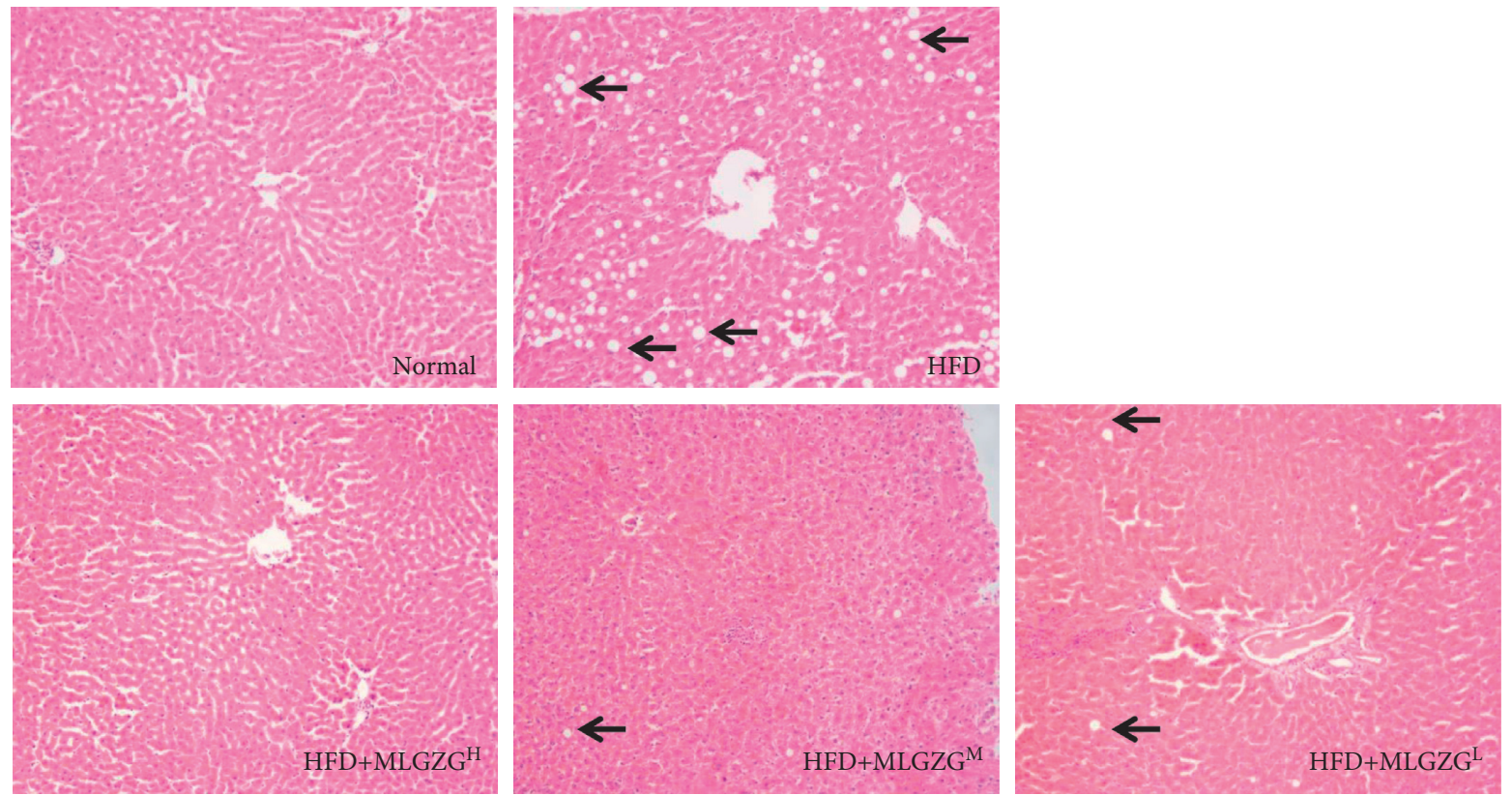

Figure 2: MLGZG ameliorates NASH in an HFD NASH rat model. Photographs of H\&E-stained paraffin sections of livers from rats treated with saline or MLGZG $(100 \times)$ (black arrow indicated hepatocyte vacuolization; HFD: high-fat diet; MLGZG ${ }^{\mathrm{L}}$ : MLGZG low dose; MLGZG ${ }^{\mathrm{M}}$ : MLGZG moderate dose; MLGZG ${ }^{\mathrm{H}}$ : MLGZG high dose).

TABLE 3: Serum lipid analysis of the rat models.

\begin{tabular}{lccccc}
\hline Group & $\mathrm{n}$ & TC $(\mu \mathrm{g} / \mathrm{L})$ & TG $(\mu \mathrm{g} / \mathrm{L})$ & HDL $(\mu \mathrm{g} / \mathrm{L})$ & LDL $(\mu \mathrm{g} / \mathrm{L})$ \\
\hline NORMAL & 10 & $1.254 \pm 0.054$ & $1.029 \pm 0.090$ & $1.223 \pm 0.057$ & $0.390 \pm 0.062$ \\
HFD & 10 & $1.693 \pm 0.052^{\#}$ & $1.415 \pm 0.074^{\#}$ & $0.772 \pm 0.650^{\#}$ & $0.751 \pm 0.065^{\#}$ \\
HFD + MLGZG $^{\mathrm{L}}$ & 10 & $1.463 \pm 0.033^{*}$ & $1.307 \pm 0.042^{*}$ & $0.883 \pm 0.082^{*}$ & $0.647 \pm 0.052^{*}$ \\
HFD + MLGZG $^{\mathrm{H}}$ & 10 & $1.340 \pm 0.033^{*}$ & $1.234 \pm 0.060^{*}$ & $0.937 \pm 0.061^{*}$ & $0.589 \pm 0.061^{*}$ \\
HFD + MLGZG $^{\mathrm{H}}$ & 10 & $1.253 \pm 0.041^{*}$ & $1.163 \pm 0.072^{*}$ & $1.105 \pm 0.052^{*}$ & $0.487 \pm 0.050^{*}$ \\
\hline
\end{tabular}

Note. Data are expressed as the mean \pm SD. TC: total cholesterol; TG: triglycerides; HDL-C: high-density lipoprotein cholesterol; LDL-C: low-density lipoprotein cholesterol. HFD: high-fat diet; MLGZG ${ }^{\mathrm{L}}$ : MLGZG low dose; MLGZG ${ }^{\mathrm{M}}$ : MLGZG moderate dose; MLGZG ${ }^{\mathrm{H}}$ : MLGZG high dose. \# indicates $P<0.05$, HFD group compared to normal control group; ${ }^{*}$ indicates $P<0.05$, MLGZG-treated groups compared to HFD group.

expression levels of the lipid genesis genes SREBP and leptin were reduced in MLGZG-treated livers (Figure 3), indicating that MLGZG is effective in protecting lipid metabolism function. Collectively, our results demonstrate that modified Lingguizhugan decoction is effective in reducing the clinical and pathological signs of NASH.

Although the mechanisms by which Lingguizhugan decoction regulates the immune response during the pathological processes of NASH are still under intense investigation, it is well established that Lingguizhugan decoction can suppress the activation of TNF-related immune responses [18]. One interesting finding from our study is that MLGZG suppresses the activation of TLR-4 and TRAF3. The role of TRAF3 in mediating the development of NASH was previously illustrated in an HFD mouse model [7]. Using both HFD- and gene knockout-induced NASH models, TLR-4/TRAF3 activation shifted immune responses and increased insulin resistance, as discussed by Wang [13]. Mitochondrial stress-induced $\mathrm{P} 65$ phosphorylation can result in NF- $\kappa$ B activation, leading to I $\kappa$ B degradation and NF- $\kappa \mathrm{B}$ nuclear translocation [19]. Researchers found that NF- $\kappa \mathrm{B}$ may be involved in an insulin resistance mechanism during hepatocyte exposure to FAs in NASH patients [26]. In this study, phosphorylated P65 upregulation was detected in NASH rats (see Figure 4), while a decrease in phosphorylated P65 was observed after MLGZG feeding. Impairment in TLR4/TRAF3 signaling can cause NF- $\kappa$ B inactivation and subsequently reduce inflammatory responses [27]. These changes played a key role in HFD-induced inflammation in NASH models, which was likely caused by the addition of MLGZG, protecting rats from hepatic injury.

Strikingly, feeding MLGZG significantly reduced the insulin resistance of NASH rats, indicating the pivotal role of modified Lingguizhugan decoction in metabolic disease therapy [16]. In our study, we found that MLGZG markedly decreased TNF- $\alpha$ expression (Figure 5). Of note, Ezquerro et al. suggested that TNF-mediated pathways play key roles in inducing apoptosis in hepatocytes [28]. Moreover, other potential modes of action include induction of the inflammatory cytokines IL- $1 \beta$ or IL- 12 by promoting signaling 


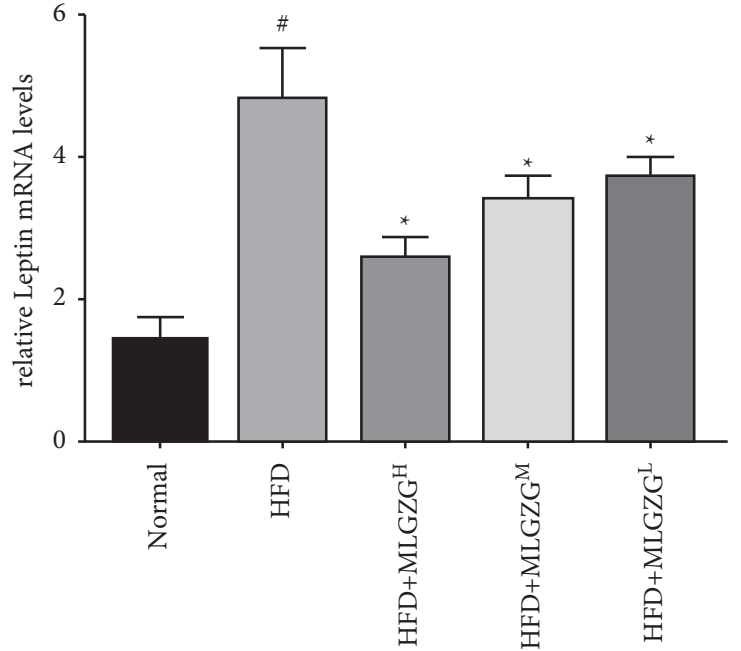

(a)

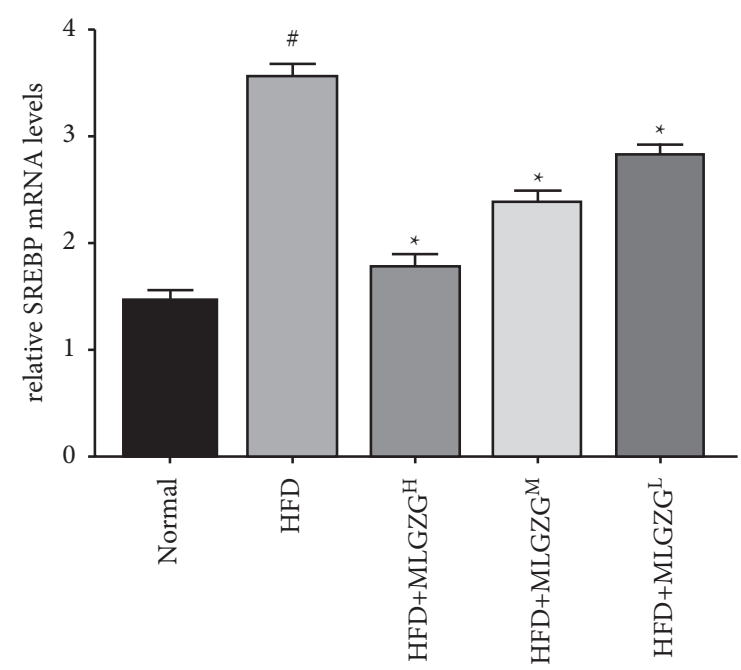

(b)

FIGURE 3: MLGZG inhibits the expression of lipid metabolic genes. Real-time PCR was used to determine the expression levels of lipid metabolic genes (a) leptin and (b) SREBP (HFD: high-fat diet; MLGZG ${ }^{\mathrm{L}}$ : MLGZG low dose; MLGZGM ${ }^{\mathrm{M}}$ MLGZG moderate dose; MLGZG $^{\mathrm{H}}$ : MLGZG high dose; \# indicates $P<0.05$, HFD group compared to the normal control group; *indicates $P<0.05$, MLGZG-treated groups compared to the HFD group).

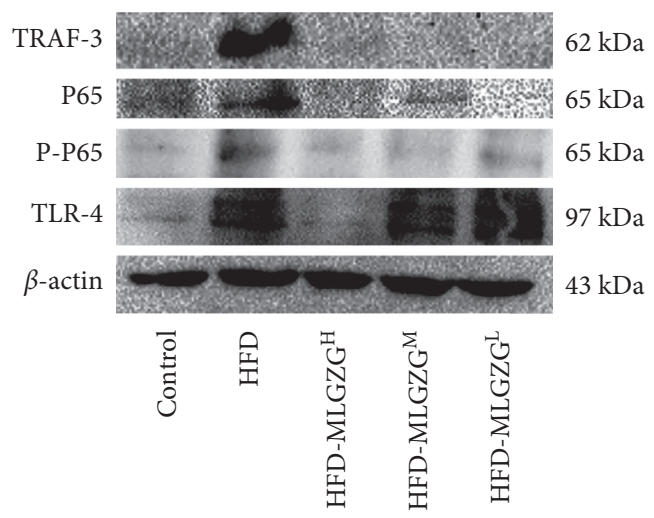

(a)

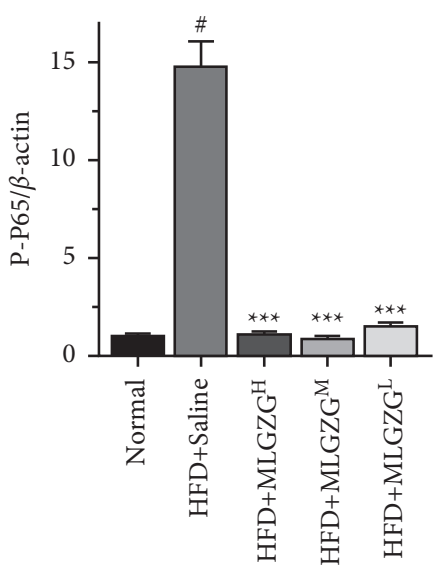

(d)

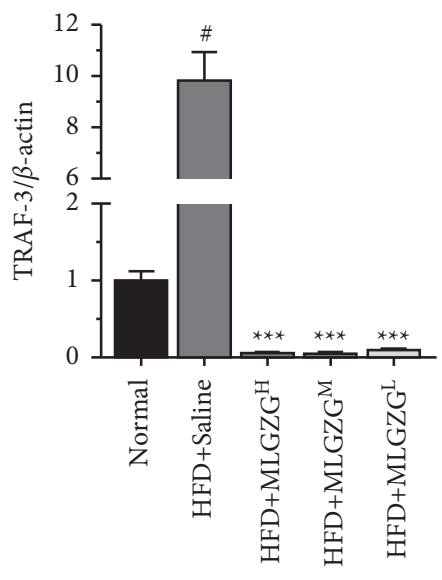

(b)

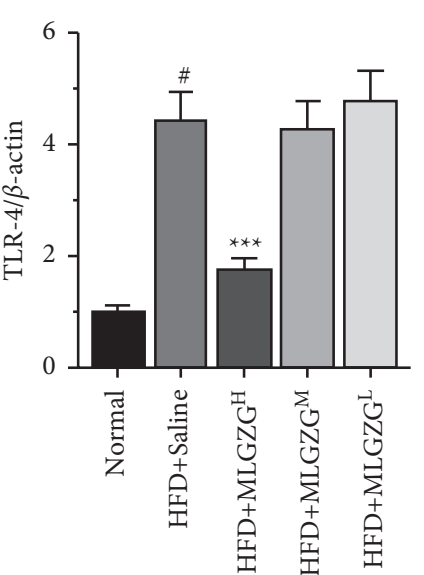

(e)

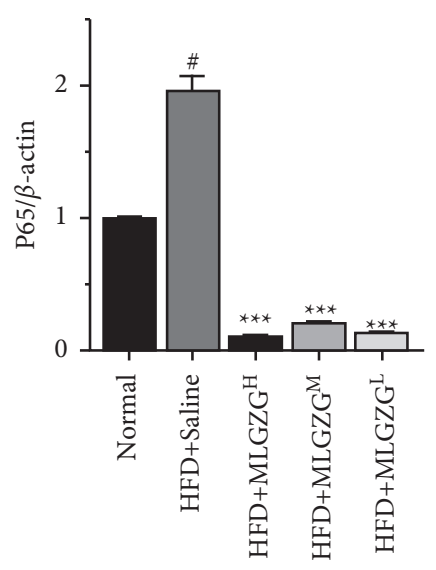

(c)

FIGURE 4: MLGZG inhibits the TLR-4/TRAF-3/NF- $\kappa$ B pathway in NASH livers. (a) Western blot analysis was conducted using whole tissue lysates from HFD-fed NASH rat livers treated with saline/MLGZG. (b-e) Bar charts represent TRAF-3/P65/P-P65/TLR-4 expression normalized against $\beta$-actin. ${ }^{*} P<0.05$ versus saline (HFD: high-fat diet; MLGZG ${ }^{\mathrm{L}}$ : MLGZG low dose; MLGZG ${ }^{\mathrm{M}}$ : MLGZG moderate dose; MLGZG $^{\mathrm{H}}$ : MLGZG high dose; \# indicates $P<0.05$, HFD group compared to the normal control group; ${ }^{* * *}$ indicates $P<0.001, \mathrm{MLGZG}-$ treated groups compared to the HFD group). 


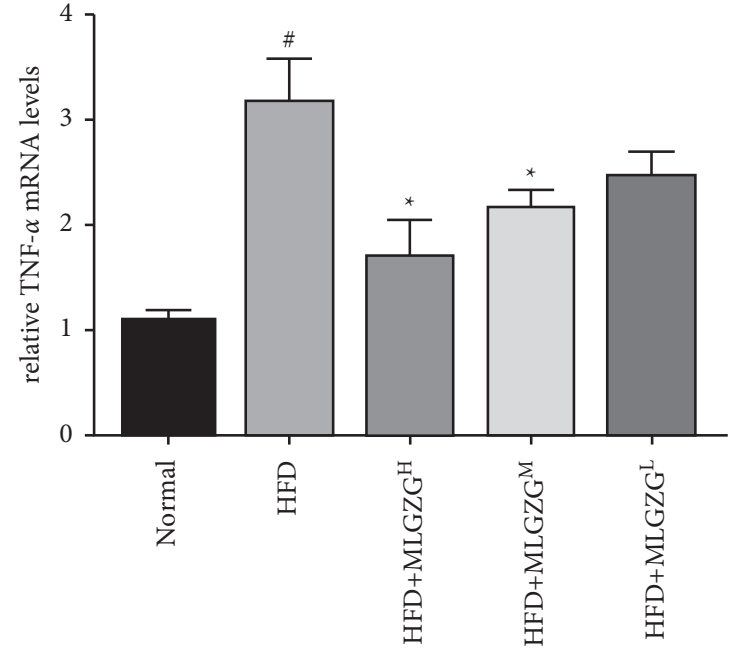

(a)

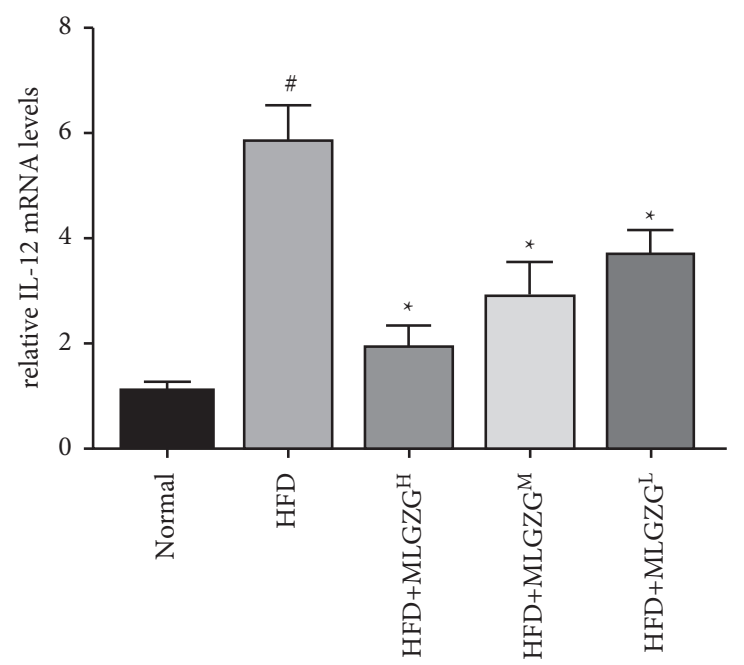

(c)

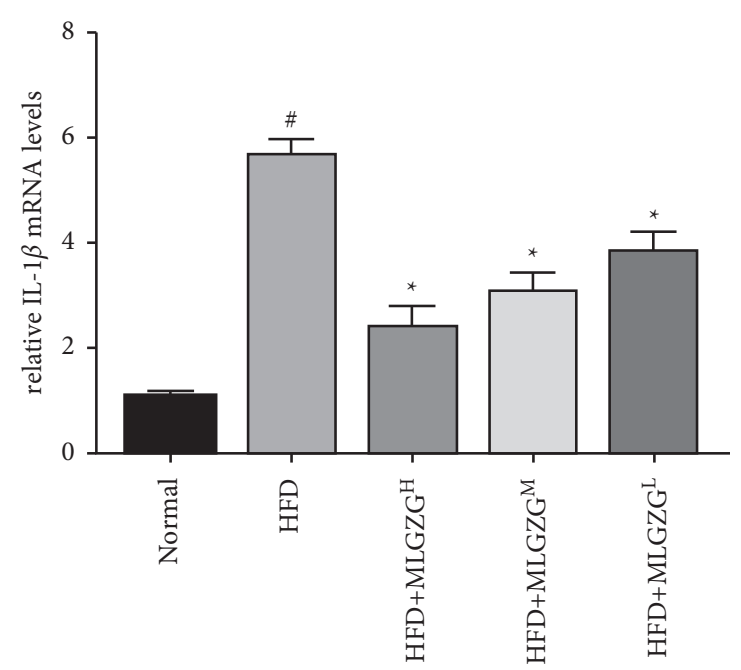

(b)

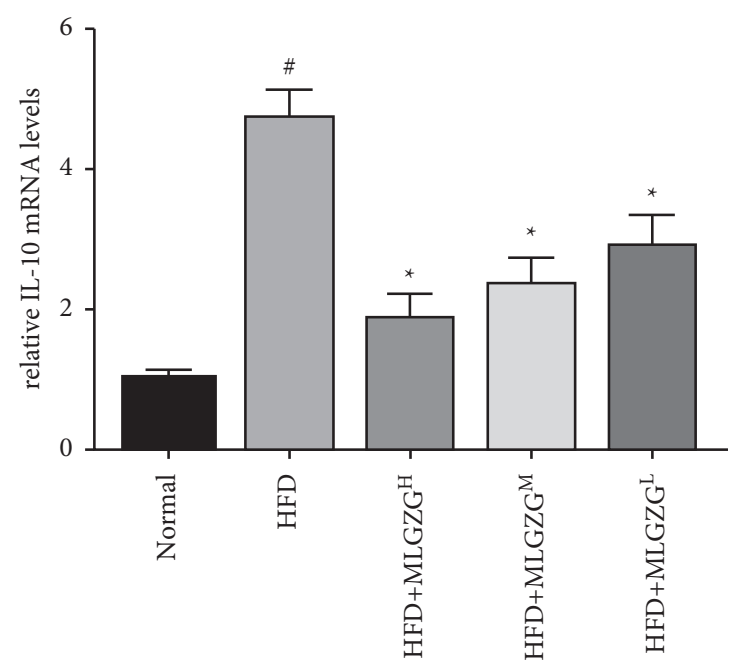

(d)

FIGURE 5: Real-time PCR was used to determine the expression levels of cytokines: (a) TNF- $\alpha$; (b) IL-1 $\beta$; (c) IL-12; and (d) IL-10 (HFD: highfat diet; MLGZGL ${ }^{\mathrm{L}}$ : MLGZG low dose; MLGZG $^{\mathrm{M}}$ : MLGZG moderate dose; MLGZG ${ }^{\mathrm{H}}$ : MLGZG high dose; \# indicates $P<0.05$, HFD group compared to the normal control group; *indicates $P<0.05$, MLGZG-treated groups compared to the HFD group).

or induction of a certain subset of T cells. In this report, we showed that MLGZG affects liver inflammatory responses by directly suppressing proinflammatory cytokine activation (Figure 5). Our results are consistent with a scenario in which reduced proinflammatory cytokines play key roles in NASH therapy in rats. A similar trend was detected by ELISA in the HFD cell model (Figure 6). Our results suggest that MLGZG decreases cytokine secretion in HFD-induced NASH livers and that the effect is related to the TLR4/ TRAF3 signaling pathway.

It has been reported that Lingguizhugan decoction can be used to treat NASH by regulating lipid genesis [29]. Hepatic TG accumulation is a key step in NASH development. Liu et al. reported that liver lipid accumulation could increase in an HFD-induced NASH model and that the use of Lingguizhugan decoction could significantly reduce lipid uptake [30]. Our study also supports their conclusion; blood TC/TG/LDL increased as the NASH model was established, but after MLGZG treatment, our results indicated that the increases in TC/TG/LDL were significantly reduced. In fact, the expression of SREBP in NASH rats was significantly lower than that in MLGZG-treated rats (see Figure 3). It should be noted that leptin expression was also reduced by MLGZG. The function of leptin is to improve the decomposition of fat; high concentrations of hepatic lipids upregulate this gene in NASH patients. We suggest that MLGZG has a protective role in NASH treatment via suppression of TLR-4-mediated inflammation activation, which subsequently reduces lipid accumulation in the liver. In view of the fact that SREBP and leptin modulate lipid genesis, it is plausible that lipid regulation genes are also involved in the enhanced therapeutic effects of MLGZG. 


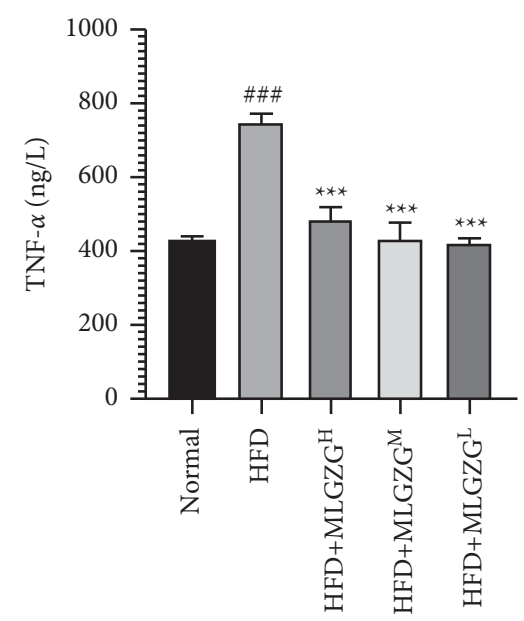

(a)

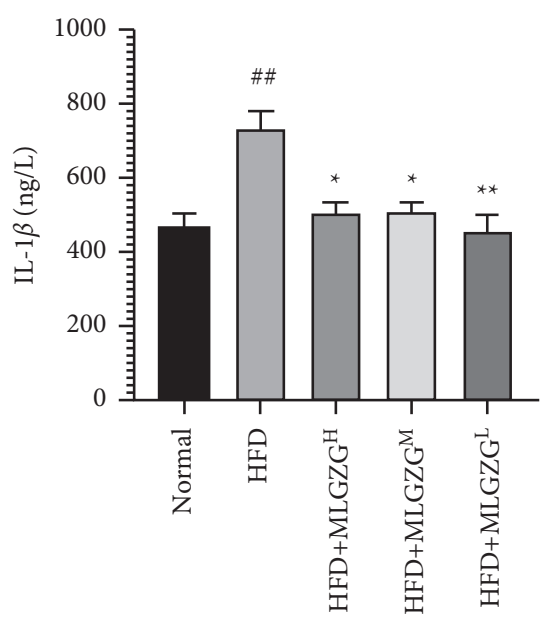

(b)

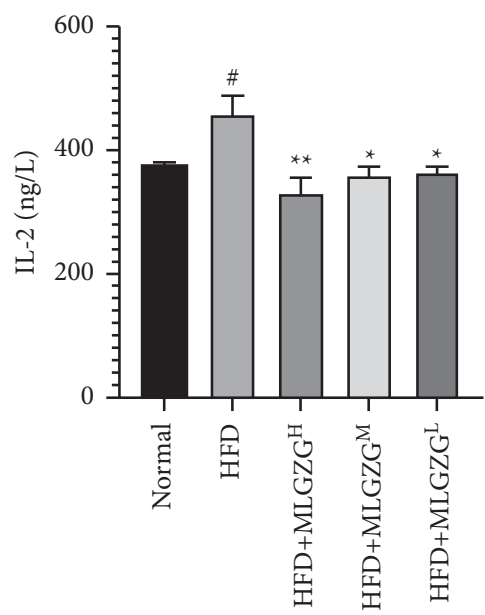

(c)

Figure 6: Effect of different doses of MLGZG on HFD HEPG2 cytokine secretion. TNF- $\alpha$, IL-1 $\beta$, and IL-2 levels in culture medium were measured by ELISA (a) TNF- $\alpha$; (b) IL- $1 \beta$; and (c) IL-2 (HFD: high-fat diet; MLGZG ${ }^{\mathrm{L}}$ : MLGZG low dose; MLGZG ${ }^{\mathrm{M}}$ : MLGZG moderate dose; MLGZG ${ }^{\mathrm{H}}$ : MLGZG high dose; \# indicates $P<0.05$, \#\# indicates $P<0.01$, and \#\#\# indicates $P<0.001$, HFD group compared to the normal control group; ${ }^{*}$ indicates $P<0.05$, ${ }^{* *}$ indicates $P<0.01$, and ${ }^{* * *}$ indicates $P<0.001$, MLGZG-treated groups compared to the HFD group).

\section{Conclusion}

Collectively, we have demonstrated that MLGZG shows immunosuppressive and therapeutic effects in treating NASH via the TLR4/TRAF3 signaling pathway. Given the critical role of TLR4/TRAF3 in regulating the immune response, it is conceivable that MLGZG might exhibit improved therapeutic efficacy in treating other metabolic diseases, such as diabetes. With satisfactory inflammation regulatory potential and few side effects, modified Lingguizhugan decoction provides a feasible approach for enhancing therapeutic efficacy in NASH patients. However, the further downstream mechanism of MLGZG treatment still needs to be investigated. Additionally, to develop more inexpensive and safer clinical applications, we need to analyze the active components of MLGZG.

\section{Abbreviations}

NAFLD: Nonalcoholic fatty liver disease

TLRs: Toll-like receptors

TLR-4: Toll-like receptor 4

NLRs: NOD-like receptors

TNF- $\alpha$ : Tumor necrosis factor alpha

RIG-I: $\quad$ Retinoic acid-inducible gene I

NF- $\kappa$ B: $\quad$ Nuclear factor kappa B

IRS1: Insulin receptor substrates 1

JNK: C-Jun n-terminal kinase

NASH: Nonalcoholic steatohepatitis

GLUT-2: Glucose transporter type 2

HFD: High-fat diet

TRAF3: TNF receptor-associated factor 3

MLGZG: Modified Lingguizhugan decoction

TNF-R: TNF receptor

SREBP: Sterol-regulatory element binding proteins

TAK-1: Transforming growth factor beta-activated kinase 1 .

\section{Data Availability}

The data used during the study are available from the corresponding author upon request.

\section{Conflicts of Interest}

The authors declare that they have no conflicts of interest.

\section{Authors' Contributions}

Jiangrong Huang and Wei Huang made equal contribution to this work.

\section{Acknowledgments}

This study was supported by the Science and Technology Research Project of Hubei Provincial Department of Education (Q20191312) and the Joint Fund of the Hubei Provincial Commission of Health and Family Planning (WJ2019-02).

\section{References}

[1] J. Y. Lee, K. H. Sohn, S. H. Rhee, and D. Hwang, "Saturated fatty acids, but not unsaturated fatty acids, induce the expression of cyclooxygenase-2 mediated through Toll-like receptor 4," Journal of Biological Chemistry, vol. 276, no. 20, pp. 16683-16689, 2001.

[2] M. T. A. Nguyen, S. Favelyukis, A.-K. Nguyen et al., "A subpopulation of macrophages infiltrates hypertrophic adipose tissue and is activated by free fatty acids via Toll-like receptors 2 and 4 and JNK-dependent pathways," Journal of Biological Chemistry, vol. 282, no. 48, pp. 35279-35292, 2007.

[3] L. Zhao, S. Zhong, H. Qu et al., "Chronic inflammation aggravates metabolic disorders of hepatic fatty acids in high-fat 
diet-induced obese mice," Scientific Reports, vol. 5, no. 1, p. 10222, 2015.

[4] M. C. Arkan, A. L. Hevener, F. R. Greten et al., "IKK- $\beta$ links inflammation to obesity-induced insulin resistance," Nature Medicine, vol. 11, no. 2, pp. 191-198, 2005.

[5] M. Monteiro-Sepulveda, S. Touch, C. Mendes-Sá et al., “Jejunal $\mathrm{T}$ cell inflammation in human obesity correlates with decreased enterocyte insulin signaling," Cell Metabolism, vol. 22, no. 1, pp. 113-124, 2015.

[6] G. Paradies, V. Paradies, F. M. Ruggiero, and G. Petrosillo, "Oxidative stress, cardiolipin and mitochondrial dysfunction in nonalcoholic fatty liver disease," World Journal of Gastroenterology, vol. 20, no. 39, pp. 14205-14218, 2014.

[7] Z. Chen, M. J. Canet, L. Sheng et al., "Hepatocyte TRAF3 promotes insulin resistance and type 2 diabetes in mice with obesity," Molecular Metabolism, vol. 4, no. 12, pp. 951-960, 2015.

[8] A. I. Lalani, C. R. Moore, C. Luo et al., "Myeloid cell TRAF3 regulates immune responses and inhibits inflammation and tumor development in mice," The Journal of Immunology, vol. 194, no. 1, pp. 334-348, 2015.

[9] P. Xie, "TRAF molecules in cell signaling and in human diseases," Journal of Molecular Signaling, vol. 8, no. 1, p. 7, 2013.

[10] X.-D. Yang and S.-C. Sun, "Targeting signaling factors for degradation, an emerging mechanism for TRAF functions," Immunological Reviews, vol. 266, no. 1, pp. 56-71, 2015.

[11] G. A. Bishop, "TRAF3 as a powerful and multitalented regulator of lymphocyte functions," Journal of Leukocyte Biology, vol. 100, no. 5, pp. 919-926, 2016.

[12] H. Häcker, P.-H. Tseng, and M. Karin, "Expanding TRAF function: TRAF3 as a tri-faced immune regulator," Nature Reviews Immunology, vol. 11, no. 7, pp. 457-468, 2011.

[13] P.-X. Wang, X.-J. Zhang, P. Luo et al., "Hepatocyte TRAF3 promotes liver steatosis and systemic insulin resistance through targeting TAK1-dependent signalling," Nature Communications, vol. 7, no. 1, p. 10592, 2016.

[14] J. Huang, X. Li, Y. Du, J. Yan, and Y. Hu, "Effects of reformatted Lingguizhugan Decoction on the glycometabolism disorder in metabolic syndrome model rats," Pharmacology and Clinics of Chinese Materia Medica, vol. 3, no. 29, p. 1679, 2013.

[15] M. Zhu, S. Hao, T. Liu et al., "Lingguizhugan decoction improves non-alcoholic fatty liver disease by altering insulin resistance and lipid metabolism related genes: a whole trancriptome study by RNA-Seq," Oncotarget, vol. 8, no. 47, pp. 82621-82631, 2017.

[16] W. Yuanyuan, J. Minghua, Z. Lina et al., "Effect of a combination of calorie-restriction therapy and Lingguizhugan decoction on levels of fasting blood lipid and inflammatory cytokines in a high-fat diet induced hyperlipidemia rat model," Journal of Traditional Chinese Medicine, vol. 35, no. 2, pp. 218-221, 2015.

[17] J. Zhou, W. Huang, and J. Huang, "HUANG xiangwu's experience on treating nonalcoholic fatty liver disease with modified Linggui Zhugan decoction," Chinese Archives of Traditional Chinese Medicine, vol. 38, no. 03, pp. 199-202, 2020.

[18] L. Yao, J. Wei, S. Shi et al., "Modified lingguizhugan decoction incorporated with dietary restriction and exercise ameliorates hyperglycemia, hyperlipidemia and hypertension in a rat model of the metabolic syndrome," BMC Complementary and Alternative Medicine, vol. 17, no. 1, p. 132, 2017.
[19] T. Zhang, J. Hu, X. Wang et al., "MicroRNA-378 promotes hepatic inflammation and fibrosis via modulation of the NF$\kappa \mathrm{B}-\mathrm{TNF} \alpha$ pathway," Journal of Hepatology, vol. 70 , no. 1 , pp. 87-96, 2019.

[20] F. Y. Yang, R. Chen, X. Zhang et al., "Preconditioning enhances the therapeutic effects of mesenchymal stem cells on colitis through PGE2-mediated T-cell modulation," Cell Transplantation, vol. 27, no. 9, pp. 1352-1367, 2018.

[21] T. Li, S. Qi, M. Unger et al., "Immuno-targeting the multifunctional CD38 using nanobody," Scientific Reports, vol. 6, no. 1, p. 27055, 2016.

[22] L. F. Iannucci, F. Cioffi, R. Senese et al., "Metabolomic analysis shows differential hepatic effects of T2 and T3 in rats after short-term feeding with high fat diet," Scientific Reports, vol. 7, no. 1, p. 2023, 2017.

[23] X. H. Du, Q. J. Chen, J. B. Song et al., "Rhubarb-aconite decoction (RAD) drug-containing serum alleviated endotoxin-induced oxidative stress injury and inflammatory response in caco-2 cells in vitro," Evidence-based Complementary and Alternative Medicine: eCAM, vol. 2020, Article ID 5834502, 2020.

[24] F.-Y. Yang, M.-X. Yu, Q. Zhou, W.-L. Chen, P. Gao, and Z. Huang, "Effects of iron oxide nanoparticle labeling on human endothelial cells," Cell Transplantation, vol. 21, no. 9, pp. 1805-1820, 2012.

[25] D. Y. Jiang, W. Q. Zhang, and J. R. Han, "Research progress of Linggui Zhugan decoction," ACTA Chinese Medicine, vol. 36, no. 12 , pp. 2562-2567, 2021.

[26] W. Chen, X. Wang, L. Huang, and B. Liu, "Hepcidin in nonalcoholic fatty liver disease regulated by the TLR4/NF- $\kappa \mathrm{B}$ signaling pathway," Experimental and Therapeutic Medicine, vol. 11, no. 1, pp. 73-76, 2016.

[27] J. Song, X. Han, Y.-L. Yao et al., "Acanthoic acid suppresses lipin $1 / 2$ via TLR4 and IRAK4 signalling pathways in EtOHand lipopolysaccharide-induced hepatic lipogenesis," Journal of Pharmacy and Pharmacology, vol. 70, no. 3, pp. 393-403, 2018.

[28] S. Ezquerro, F. Mocha, G. Frühbeck et al., "Ghrelin reduces TNF- $\alpha$-induced human hepatocyte apoptosis, autophagy and pyroptosis: role in obesity-associated NAFLD," Journal of Clinical Endocrinology \& Metabolism, vol. 104, no. 1, pp. 21-37, 2019.

[29] Y. Yang, Q. Li, S. Chen, B. Ke, Y. Huang, and J. Qin, "Effects of modified lingguizhugan decoction combined with weekend fasting on metabolic syndrome," Journal of Traditional Chinese Medicine, vol. 34, no. 1, pp. 48-51, 2014.

[30] T. Liu, L.-L. Yang, L. Zou et al., "Chinese medicine formula lingguizhugan decoction improves Beta-oxidation and metabolism of Fatty Acid in high-fat-diet-induced rat model of Fatty liver disease," Evidence-based Complementary and Alternative Medicine, vol. 2013, pp. 1-9, 2013. 